\title{
Condição de contorno para calibração de reflectômetro usado para medição de água no solo
}

\author{
Boundary conditions for calibration of reflectometer used to measure soil water
}

\author{
Paulo Ivonir Gubiani ${ }^{* *}$ Dalvan José Reinert ${ }^{\mathrm{I}}$ José Miguel Reichert $^{\mathrm{I}}$ \\ Neiva Somavila Gelain"
}

\section{RESUMO}

A reflectometria no domínio do tempo (TDR, na sigla em inglês) é uma técnica indireta usada para estimar o conteúdo de água do solo $(\theta)$ em função de sua constante dielétrica aparente $\left(K_{d}\right)$. Neste estudo, o objetivo principal foi investigar as consequências de se incluir ou não medidas de $K_{a}$ em água, na elaboração da curva de calibração do TDR. Foram avaliados horizontes de solo com $0,13 \mathrm{~kg} \mathrm{kg-1}^{-1}, 0,23 \mathrm{~kg} \mathrm{~kg}^{-1}$ e 0,64kg kg-1 de argila. Para cada horizonte, a $K_{a}$ foi medida em amostras desde solo seco até solo saturado. Adicionalmente, a $K_{a}$ foi medida apenas em água. As medições foram feitas usando-se cabos coaxiais RGC58, 50Ohm e sensor dupla haste metálica de $19 \mathrm{~cm}$. Para cada horizonte, foram formados dois conjuntos de dados com $\theta$ e $K_{a}$, um com e outro sem as medidas

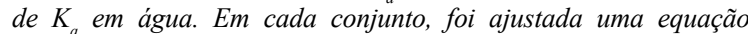
polinomial de terceiro grau, estimando $\theta$ em função de $K_{a}$. Com a inclusão das medidas de $K$ em água, o polinômio de terceiro grau se ajustou melhor ao extremo saturado e preveniu erros na estimativa de $\theta$. Como não há garantia de que as medidas de $K$ de campo não serão maiores que as medidas de $K_{a}$ das amostras saturadas em laboratório, recomenda-se a inclusão de medidas de $K_{a}$ em água para obtenção da curva de calibração. Sem esse procedimento e sem verificação de campo das medidas de $K_{a^{\prime}}$ erros na estimativa de $\theta$ da ordem de $0,1 \mathrm{~m}^{3} \mathrm{~m}^{-3}$ podem ocorrer facilmente.

Palavras-chave: medição de água no solo, reflectometria, constante dielétrica.

\section{ABSTRACT}

The time domain reflectometry is a technique used to estimate soil water content $(\theta)$ as a function of its apparent dielectric constant $\left(K_{d}\right)$. In this study the main objective was to investigate the consequences of including or not measures of $K_{a}$ in water on the TDR calibration curve. Three horizons with different clay content were used: $0.13 \mathrm{~kg} \mathrm{~kg}^{-1}, 0.23 \mathrm{~kg} \mathrm{~kg}^{-1}$ and $0.64 \mathrm{~kg} \mathrm{~kg}^{-1}$. In each horizon, $K_{a}$ was measured from dry to saturated soil. Additionally, $K_{a}$ was measured in water. Measurements were made using coaxial cables RGC58, 50Ohms and double stainless rod of $19 \mathrm{~cm}$. For each soil two data sets with $\theta$ and $K_{a}$ were formed, one with and another without the addition of $K$ measured in water. In each set a cubic polynomial equation was adjusted, estimating $\theta$ as a function of $K_{a}$. With the inclusion of $K_{a}$ measured in water the cubic polynomial adjusted better the extreme saturated and avoided errors in the estimated $\theta$. Since there is no certainty that the measures of $K_{a}$ on field will not be greater than the measures of $K_{a}$ in the saturated samples in the laboratory, it's recommend to include measures of $K_{a}$ in water to obtain the calibration curve. Without this procedure and no field verification of $K_{a}$ measurements, errors in estimated $\theta$ of the order of $0.1 \mathrm{~m}^{3} \mathrm{~m}^{-3}$ can easily occur.

Key words: measurement of soil water, reflectometry, dielectric constant.

\section{INTRODUÇÃO}

O monitoramento do conteúdo de água no solo é necessário em estudos sobre a dinâmica da água no solo e para planejamento do uso racional da água nas atividades agrícolas. Métodos diretos de medição do conteúdo de água por meio da coleta de amostras de solo são laboriosos e destrutivos, o que inviabiliza estudos detalhados sobre a dinâmica da água no solo. Diferentemente, os métodos indiretos não destrutivos são, na maioria, automatizados e fornecem medidas detalhadas do conteúdo de água no solo no espaço e no tempo.

Entre os métodos indiretos, a reflectometria no domínio do tempo (TDR, na sigla em inglês) é um

'Departamento de Solos, Centro de Ciências Rurais (CCR), Universidade Federal de Santa Maria (UFSM), 97105-900, Santa Maria, RS,

Brasil. E-mail: paulogubiani@gmail.com. *Autor para correspondência.

IIAgrosul Assessoria Agronômica, Itaqui, RS, Brasil. 
método bastante usado no monitoramento do conteúdo de água no solo. Conforme descrito em JONES et al. (2002), a aplicação dessa técnica em solos baseia-se na mudança da constante dielétrica aparente do solo $\left(\mathrm{K}_{\mathrm{a}}\right)$, causada pela mudança no conteúdo de água. Essa interferência é capturada pelo TDR por meio de medidas da velocidade de propagação de um pulso elétrico em guias metálicas inseridas no solo, onde a velocidade é inversamente proporcional ao valor de $\mathrm{K}_{\mathrm{a}}$. $\mathrm{O}$ valor de $\mathrm{K}_{\mathrm{a}}$ de um solo seco fica entre 3 e 5 , dependendo de sua composição mineralógica e orgânica, enquanto que a água pura tem valor de $\mathrm{K}_{\mathrm{a}}$ em torno de 81 .

À medida que aumenta o conteúdo volumétrico de água no solo $\left(\theta, \mathrm{m}^{3} \mathrm{~m}^{-3}\right)$, a $\mathrm{K}_{\mathrm{a}}$ (adimensional) também aumenta. A relação entre $\theta$ e $K_{a}$ é conhecida como curva de calibração, a qual possibilita estimar $\theta$ em função de $\mathrm{K}_{\mathrm{a}}$. Normalmente, os TDR para medição da $\mathrm{K}_{\mathrm{a}}$ em solos já fornecem de fábrica uma curva de calibração genérica, a equação proposta por TOPP et al. (1980). Porém, a curva de calibração genérica fornecida pelo fabricante pode não estimar adequadamente o $\theta$ (TOMAZELLI \& BACCHI, 2001; COELHO et al., 2006; ELAIUY et al. 2009; KAISER et al., 2010; SANTOS et al., 2010).

Em alguns casos, há necessidade ainda de corrigir a calibração obtida em laboratório com medidas posteriores de campo (STANGL et al., 2009), procedimento que pode ser feito individualmente por guia de TDR (SERRARENS et al., 2000). O aumento no comprimento do cabo entre o emissor do pulso elétrico e a guia de medição instalada no campo pode atenuar o sinal refletido e alterar o valor de $\mathrm{K}_{\mathrm{a}}$ (COELHO et al., 2005). Assim, a obtenção de curva de calibração específica para cada solo e comprimento de cabo usado no campo é uma prática recomendada para melhorar a acurácia da estimativa de $\theta$.

Para obtenção de uma curva de calibração representativa, a $\mathrm{K}_{\mathrm{a}}$ deve ser medida desde solo com mínimo (solo praticamente sem água) até máximo $\theta$ (solo saturado). Além disso, a inclusão no conjunto de dados da $\mathrm{K}_{\mathrm{a}}$ medida apenas em água fornece uma condição de contorno para o ajuste da equação de calibração que previne erros de estimativa no campo, principalmente para $\theta$ próximos da saturação.

Devido à incapacidade de representar toda a variabilidade da estrutura do solo do campo nas amostras usadas para a calibração, a $\mathrm{K}_{\mathrm{a}}$ medida em solo saturado no campo pode ser maior que a $\mathrm{K}_{\mathrm{a}}$ medida nas amostras de solo saturadas usadas para a calibração. Por isso, é importante garantir que a extrapolação da curva de calibração estime conteúdos de água que sigam uma tendência coerente em direção à $\mathrm{K}_{\mathrm{a}}$ medida apenas em água. Assim, o objetivo deste trabalho é investigar a interferência da inclusão de medidas de $\mathrm{K}_{\mathrm{a}}$ em água na curva de calibração do TDR100.

\section{MATERIAL E MÉTODOS}

A curva de calibração do TDR100 foi obtida para a camada de solo de $0-0,8 \mathrm{~m}$ do horizonte A e da camada de 1,5-2,0m do horizonte Bt de um Argissolo, respectivamente, denominados de Argissolo-A $\left(0,13 \mathrm{~kg} \mathrm{~kg}^{-1}\right.$ de argila, $0,10 \mathrm{~kg} \mathrm{~kg}^{-1}$ de silte e $0,77 \mathrm{~kg} \mathrm{~kg}^{-1}$ de areia) e Argissolo-Bt $(0,23 \mathrm{~kg}$ $\mathrm{kg}^{-1}$ de argila, $0,13 \mathrm{~kg} \mathrm{~kg}^{-1}$ de silte e $0,64 \mathrm{~kg} \mathrm{~kg}^{-1}$ de areia) e da camada de $0-0,3 \mathrm{~m}$ do horizonte A de um Latossolo, denominado de Latossolo-A $\left(0,64 \mathrm{~kg} \mathrm{~kg}^{-1}\right.$ de argila, $0,24 \mathrm{~kg} \mathrm{~kg}^{-1}$ de silte e $0,12 \mathrm{~kg} \mathrm{~kg}^{-1}$ de areia).

Em cada horizonte, aproximadamente $50 \mathrm{~kg}$ de solo foi coletado, colocado para secar ao ar e passado por peneira de $2 \mathrm{~mm}$, obtendo-se a terra fina seca ao ar (TFSA). O conteúdo gravimétrico de água da TFSA foi quantificado em estufa a $105^{\circ} \mathrm{C}$ por 24 horas. Na sequência, quatro amostras de $3,5 \mathrm{~kg}$ da TFSA foram colocadas na estufa a $105^{\circ} \mathrm{C}$. A mudança de massa dessas amostras foi monitorada ao longo do tempo, a fim de se obter amostras com conteúdos de água diferentes entre si e menores que o conteúdo de água da TFSA. Em várias outras amostras de $3,5 \mathrm{~kg}$ da TFSA, água foi adicionada de modo a aumentar gradativamente o seu conteúdo até a condição de umidade que ainda possibilitava a homogeneização da amostra. Devido ao aumento da retenção de água com o aumento no teor de argila, o número de amostras foi crescente na sequência Argissolo-A (10 amostras), Argissolo-B (11 amostras) e Latossolo-A (15 amostras), a fim de se manter um intervalo de aproximadamente $0,05 \mathrm{~m}^{3} \mathrm{~m}^{-3}$ entre uma amostra e outra.

As amostras foram transferidas para colunas de PVC, com diâmetro de $10 \mathrm{~cm}$ e altura de $30 \mathrm{~cm}$. A quantidade de solo transferida e acondicionada nas colunas de PVC (em camadas de $2 \mathrm{~cm}$ de espessura) foi calculada para que as amostras ficassem com densidade mais próxima da densidade de campo $\left(1590 \mathrm{~kg} \mathrm{~m}^{-3}\right.$ para o Argissolo-A, $1555 \mathrm{~kg} \mathrm{~m}^{-3}$ para o Argissolo-Bt e $1300 \mathrm{~kg} \mathrm{~m}^{-3}$ para o Latossolo-A). Também foi preparada uma amostra de solo saturado numa coluna de PVC e outra coluna de PVC contendo apenas água.

No centro de cada coluna de PVC foi inserida verticalmente uma sonda de TDR, construída com duas hastes de aço inoxidável de $0,5 \mathrm{~cm}$ de diâmetro e $19 \mathrm{~cm}$ de comprimento. O corpo de fixação 
da sonda foi construído de resina epóxi, que manteve o paralelismo das guias espaçadas $4,5 \mathrm{~cm}$.

A constante dielétrica das amostras foi medida com diferentes comprimentos de cabo. Para o Argissolo, foi usado cabo coaxial RGC58, 50Ohm, com comprimento de 3, 10 e $25 \mathrm{~m}$. Para o Latossolo, foi usado apenas o cabo de $3 \mathrm{~m}$, porque não se tinha evidências de efeito do comprimento do cabo e porque a calibração para o Latossolo foi feita em período anterior ao da calibração para o Argissolo. Após indícios de campo, com o uso da curva de calibração do Latossolo, de que poderia haver efeito do comprimento do cabo, optou-se por investigar esse fator no Argissolo.

As sondas foram conectadas a um multiplexador SDMX50, ligado ao TDR100, e este a um datalogger CR10X para o armazenamento dos dados (Campbell Scientific, Inc.). Em cada amostra, a constante dielétrica ( $\mathrm{K}_{\mathrm{a}}$, adimensional) foi medida no mínimo cinco vezes. O conteúdo de água de cada amostra foi expresso em base volumétrica $\left(\theta, \mathrm{m}^{3} \mathrm{~m}^{-3}\right)$. Aos dados medidos foi ajustado o modelopolinomial de terceiro grau: $\quad \theta=\mathrm{a}+\mathrm{bK}_{\mathrm{a}}+\mathrm{cK}_{\mathrm{a}}^{2}+\mathrm{dK}_{\mathrm{a}}^{3}$ em que $a, b, c$ e d são os parâmetros de ajuste do modelo, respectivamente, para o termo constante, de primeiro, segundo e terceiro grau.

A equação (1) foi ajustada com e sem as medidas de $\mathrm{K}_{\mathrm{a}}$ feitas em água, por análise de regressão, e a significância dos parâmetros do modelo foi avaliada pelo teste t a 5\%. As equações de calibração obtidas foram comparadas com a equação polinomial de terceiro grau obtida por TOPP et al. (1980), na qual o valor dos parâmetro são: $\mathrm{a}=-5,3 \cdot 10^{-2} ; \mathrm{b}=2,92 \cdot 10^{-2}$; $c=-5,5 \cdot 10^{-4} ; d=-4,3 \cdot 10^{-6}$.

\section{RESULTADOS E DISCUSSÃO}

$\mathrm{O}$ número de amostras que pode ser preparado foi diferente para cada solo, devido às diferenças do intervalo de conteúdo de água entre solo seco e solo saturado $\left(\theta_{\mathrm{s}}\right)$ (Tabela 1$)$. O $\theta_{\mathrm{s}}$ aumentou na sequência Argissolo-A $\left(0,44 \mathrm{~m}^{3} \mathrm{~m}^{-3}\right)$, Argissolo-B $\left(0,45 \mathrm{~m}^{3} \mathrm{~m}^{-3}\right)$ e Latossolo-A $\left(0,58 \mathrm{~m}^{3} \mathrm{~m}^{-3}\right)$, na mesma sequência do aumento no teor de argila $(0,13,0,23$, e, $0,64 \mathrm{~kg} \mathrm{~kg}^{-1}$, respectivamente).

$\mathrm{Na}$ amostra de solo seco em estufa a $105^{\circ} \mathrm{C}$ por $24 \mathrm{~h}$ do Latossolo-A, o sinal de reflexão do pulso elétrico não teve resolução suficiente para a medição da $\mathrm{K}_{\mathrm{a}}$ pelo TDR100. Excluindo-se essa amostra, a $\mathrm{K}_{\mathrm{a}}$ média ficou em torno de 3,5 para todos os solos na condição de mínimo $\theta$, mas, na saturação, ela foi bem diferente entre os solos (27,39 no Argissolo-A; 35,60 no Argissolo-B e 42,50 no Latossolo-A) devido às diferenças no $\theta_{\mathrm{s}}$ (Tabela 1 ).

No Latossolo-A, o desvio padrão da $\mathrm{K}_{\mathrm{a}}$ foi pouco alterado em toda a faixa de $\theta$ (Tabela 1). Diferentemente, no Argissolo-A e no Argissolo-B, houve aumento do desvio padrão da $\mathrm{K}_{\mathrm{a}}$ com $\mathrm{o}$ aumento de $\theta$, indicando aumento da variabilidade de $\mathrm{K}_{\mathrm{a}}$ por efeito do comprimento dos cabos.

A equação (1) ajustou-se bem aos dados medidos, pois o $\mathrm{R}^{2}$ foi maior que 0,97 para todos os

Tabela 1 - Médias e desvio padrão da constante dielétrica $\left(\mathrm{K}_{\mathrm{a}}\right)$ para cada conteúdo de água $(\theta)$ da série de amostras de cada solo.

\begin{tabular}{|c|c|c|c|c|c|}
\hline$\theta, \mathrm{m}^{3} \mathrm{~m}^{-3}$ & $\mathrm{~K}_{\mathrm{a}}$ & $\theta, \mathrm{m}^{3} \mathrm{~m}^{-3}$ & $\mathrm{~K}_{\mathrm{a}}$ & $\theta, \mathrm{m}^{3} \mathrm{~m}^{-3}$ & $\mathrm{~K}_{\mathrm{a}}$ \\
\hline 0,00 & $3,75(0,19)$ & 0,00 & $3,34(0,07)$ & 0,01 & $3,85(0,01)$ \\
\hline 0,02 & $4,03(0,24)$ & 0,04 & $4,8(0,35)$ & 0,12 & $5,35(0,02)$ \\
\hline 0,05 & $4,54(0,11)$ & 0,09 & $5,68(0,19)$ & 0,13 & $6,25(0,03)$ \\
\hline 0,10 & $6,94(0,17)$ & 0,11 & $5,96(0,17)$ & 0,17 & $6,95(0,03)$ \\
\hline 0,17 & $12,20(0,37)$ & 0,14 & $6,46(0,17)$ & 0,21 & $8,39(0,01)$ \\
\hline 0,22 & $15,83(0,58)$ & 0,15 & $8,24(0,22)$ & 0,22 & $10,50(0,04)$ \\
\hline 0,26 & $17,92(0,36)$ & 0,17 & $10,37(0,33)$ & 0,27 & $13,88(0,04)$ \\
\hline 0,31 & $20,74(0,73)$ & 0,24 & $12,89(0,49)$ & 0,31 & $17,30(0,05)$ \\
\hline 0,44 & $27,39(1,01)$ & 0,30 & $16,72(0,82)$ & 0,34 & $21,39(0,05)$ \\
\hline 1,00 & $83,56(1,27)$ & 0,45 & $35,60(1,81)$ & 0,39 & $27,19(0,3)$ \\
\hline- & - & 1,00 & $84,78(1,31)$ & 0,41 & $33,54(0,09)$ \\
\hline- & - & - & - & 0,45 & $35,54(0,12)$ \\
\hline- & - & - & - & 0,47 & $35,17(0,25)$ \\
\hline- & - & - & - & 0,58 & $42,50(0,23)$ \\
\hline- & - & - & - & 1,00 & $80,94(0,03)$ \\
\hline
\end{tabular}

${ }^{(1)}$ As medidas de $\mathrm{K}_{\mathrm{a}}$ foram feitas com cabos coaxiais $50 \mathrm{Ohm}$ de 3,10 e $25 \mathrm{~m}$. ${ }^{(2)}$ As medidas de $\mathrm{K}_{\mathrm{a}}$ foram feitas somente com cabos coaxiais $50 \mathrm{Ohm}$ de $3 \mathrm{~m}$. Números entre parênteses representam o desvio padrão. 
ajustes. Porém, em seis dos 14 ajustes (Argissolo-A-3-c, Argissolo-A-10-c, Argissolo-A-25-c, Argissolo-Bt-3-c, Argissolo-Bt-10-c e Argissolo-Bt-25-c), um ou mais parâmetros de ajuste não tiveram efeito significativo no modelo (Tabela 2). Apesar disso, optou-se pela manutenção de todos os parâmetros da equação (1), o que proporcionava aumento do coeficiente de determinação $\mathrm{R}^{2}$, indicando melhoria na estimativa do modelo. Outro motivo foi a decisão de se obter equações com todos os termos até o terceiro grau, para compará-las com a equação sugerida por TOPP et al. (1980), que também tem todos os termos até o terceiro grau.

Com a inclusão das medidas de $\mathrm{K}_{\mathrm{a}}$ em água, as equações obtidas geraram curvas que acompanharam a tendência da curva definida pela equação de TOPP et al. (1980) em toda a faixa de $\mathrm{K}_{\mathrm{a}}$ (Figura 1). Diferentemente, sem a inclusão das medidas de $\mathrm{K}_{\mathrm{a}}$ em água, as equações geraram curvas que iniciaram desvio expressivo em relação à curva definida pela equação de TOPP et al. (1980), a partir de valores de $\mathrm{K}_{\mathrm{a}}$ em torno de 30 (Figura 1), que são menores que os $\mathrm{K}_{\mathrm{a}}$ medidos em solo saturado para o Argissolo-B e Latossolo-A (Tabela 1).

$\mathrm{A}$ inclusão de medidas de $\mathrm{K}_{\mathrm{a}}$ em água é aconselhável, mesmo para curvas de uso restrito ao solo para o qual ela é elaborada. Caso as medidas da $\mathrm{K}_{\mathrm{a}}$ em solo próximo à saturação no campo forem maiores que a $\mathrm{K}_{\mathrm{a}}$ da amostra saturada da qual se obteve a curva de calibração, o uso da curva de calibração sem a condição de contorno pode resultar superestimativa (Figuras 1b e 1f) ou subestimativa (Figura 1d) do conteúdo de água no solo. Essas diferenças podem aumentar bruscamente a partir de $\mathrm{K}_{\mathrm{a}}=27$ no Argissolo-A, $\mathrm{K}_{\mathrm{a}}=37$ no Argissolo-Bt e $\mathrm{K}_{\mathrm{a}}=40$ no Latossolo-A (Figura 2), que são quase todos menores que os valores de $\mathrm{K}_{\mathrm{a}}$ medidos nas amostras saturadas (Tabela 1).

Para os três solos usados na calibração, o erro no conteúdo de água estimado, incluindo possíveis efeitos do comprimento de cabo e da obtenção de curvas de calibração sem a inclusão de medidas de $\mathrm{K}_{\mathrm{a}}$ em água, pode ser maior que $0,1 \mathrm{~m}^{3} \mathrm{~m}^{-3}$, se o TDR100 medir, no campo, $\mathrm{K}_{\mathrm{a}}$ com 5 unidades a mais que a $\mathrm{K}_{\mathrm{a}}$ medida no solo saturado no laboratório (Figura 2). Isso pode acontecer se o solo no campo onde a sonda de TDR é inserida estiver saturado e sua porosidade total for maior que a porosidade total da amostra usada na calibração. Tendo em vista que a condição de solo saturado não é a mais frequente no campo, a ocorrência e a magnitude desses erros podem ser pequenas. Para que elas sejam quantificadas, é necessário uma análise de séries de medições de campo.

O uso de curva de calibração sem condição de contorno (sem medidas de $\mathrm{K}_{\mathrm{a}}$ em água) requer maior atenção com as estimativas do $\theta$ em solo próximo da saturação e aumenta a chance de necessidade de correção da curva de calibração, procedimento recomendado por SERRARENS et al. (2000) e STANGL et al. (2009). Portanto, a validade da curva é restrita para o intervalo de $\mathrm{K}_{\mathrm{a}}$ medidos no campo, que permanecem dentro do intervalo de $\mathrm{K}_{\mathrm{a}}$ usados na curva de calibração.

Cabe ressaltar que o uso de curva decalibração, incluindo medidas de $\mathrm{K}_{\mathrm{a}}$ em água não

Tabela 2 - Valores estimados dos parâmetros da equação (1) ajustada com e sem condição de contorno para as diferentes combinações de solo e comprimentos de cabo.

\begin{tabular}{lcccc}
\hline Combinação $^{(1)}$ & $\mathrm{a}$ & $\mathrm{b}$ & $\mathrm{c}$ & $\mathrm{d}$ \\
\hline Argissolo-A-3-c & $-4,4430 \mathrm{E}-02$ & $1,7360 \mathrm{E}-02$ & $0,68780 \mathrm{E}-04$ & $-1,5300 \mathrm{E}-06$ \\
Argissolo-A-10-c & $-5,1720 \mathrm{E}-02$ & $1,7540 \mathrm{E}-02$ & $288,00 \mathrm{E}-04$ & $-0,74665 \mathrm{E}-06$ \\
Argissolo-A-25-c & $-4,1930 \mathrm{E}-02$ & $1,6830 \mathrm{E}-02$ & $22,350 \mathrm{E}-04$ & $-0,89448 \mathrm{E}-06$ \\
Argissolo-A-3-s & $-10,484 \mathrm{E}-02$ & $3,7810 \mathrm{E}-02$ & $-16,000 \mathrm{E}-04$ & $36,290 \mathrm{E}-06$ \\
Argissolo-A-10-s & $-11,916 \mathrm{E}-02$ & $3,8660 \mathrm{E}-02$ & $-15,900 \mathrm{E}-04$ & $32,880 \mathrm{E}-06$ \\
Argissolo-A-25-s & $-9,8970 \mathrm{E}-02$ & $3,4860 \mathrm{E}-02$ & $-13,400 \mathrm{E}-04$ & $27,720 \mathrm{E}-06$ \\
Argissolo-Bt-3-c & $-7,4950 \mathrm{E}-02$ & $3,2770 \mathrm{E}-02$ & $-6,6973 \mathrm{E}-04$ & $5,1500 \mathrm{E}-06$ \\
Argissolo-Bt-10-c & $-6,2420 \mathrm{E}-02$ & $2,9470 \mathrm{E}-02$ & $-5,7684 \mathrm{E}-04$ & 0,9970 \\
Argissolo-Bt-25-c & $-4,0200 \mathrm{E}-02$ & $2,6360 \mathrm{E}-02$ & $-4,6996 \mathrm{E}-04$ & 0,9946 \\
Argissolo-Bt-3-s & $-8,4260 \mathrm{E}-02$ & $3,4450 \mathrm{E}-02$ & $-7,0863 \mathrm{E}-04$ & $3,5900 \mathrm{E}-06$ \\
Argissolo-Bt-10-s & $-4,1160 \mathrm{E}-02$ & $2,4160 \mathrm{E}-02$ & $-2,2727 \mathrm{E}-04$ & $4,8600 \mathrm{E}-06$ \\
Argissolo-Bt-25-s & $-0,65100 \mathrm{E}-02$ & $1,7920 \mathrm{E}-02$ & $0,97120 \mathrm{E}-04$ & $-1,6900 \mathrm{E}-06$ \\
Latossolo-A-3-c & $1,9170 \mathrm{E}-02$ & $2,1250 \mathrm{E}-02$ & $-6,9200 \mathrm{E}-06$ & 0,9948 \\
Latossolo-A-3-s & $-8,6740 \mathrm{E}-02$ & $4,4300 \mathrm{E}-02$ & $-3,4048 \mathrm{E}-04$ & 0,9831 \\
\hline
\end{tabular}

(1) A sequência de informações representa o solo (Argissolo e Latossolo), o horizonte (A e $\mathrm{Bt}$ ), o comprimento do cabo (3, 10 e 25m) e a condição de ajuste da equação (1) com (c) e sem (s) as medidas de $\mathrm{K}_{\mathrm{a}}$ em água. 


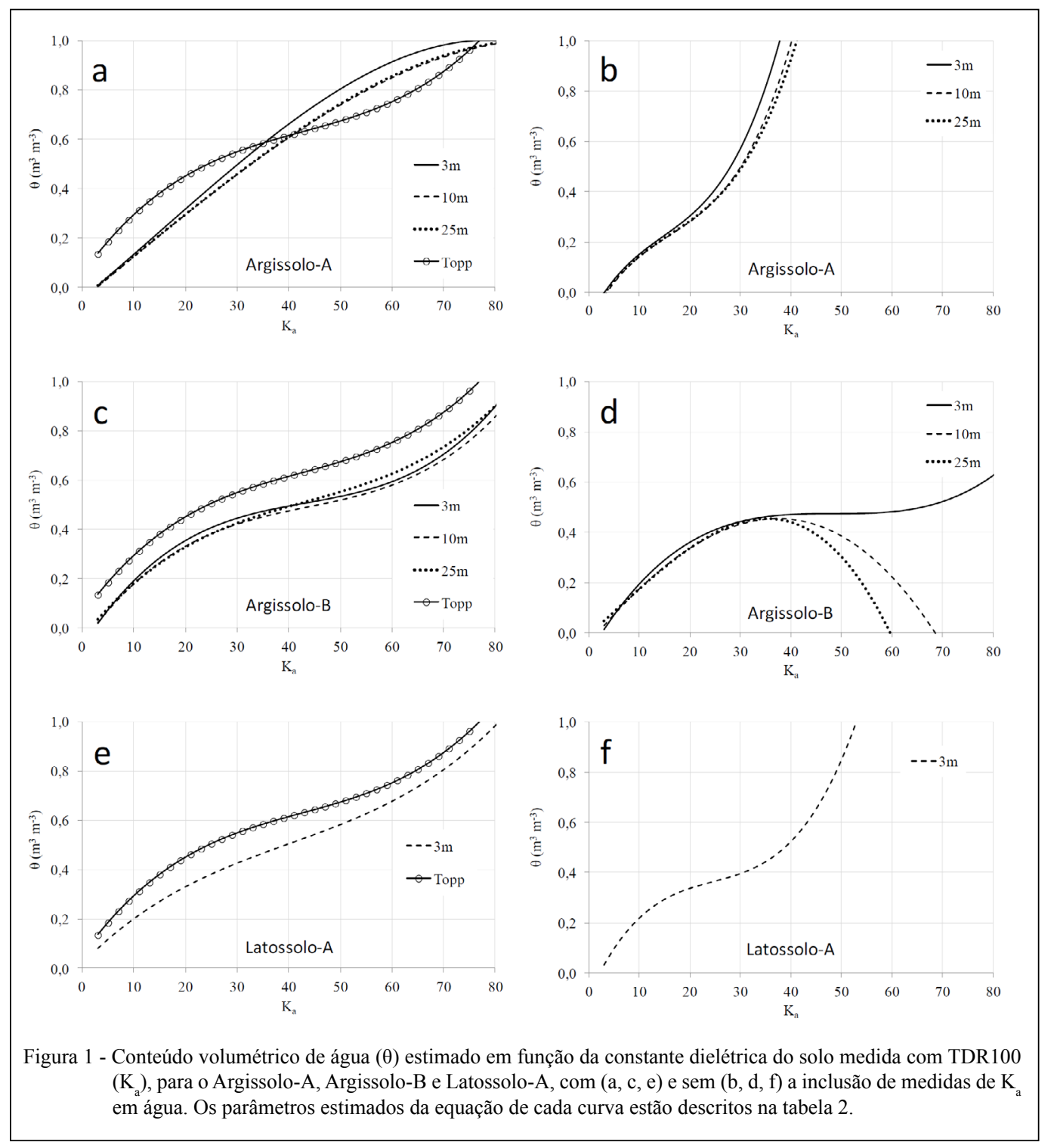

dispensa a verificação de campo, nem garante que a curva obtida com a equação (1) será concordante com a de TOOP et al. (1980), fato observado neste estudo (Figura 1) e já conhecido de estudos anteriores (TOMAZELLI \& BACCHI, 2001; COELHO et al., 2006; ELAIUY et al. 2009; KAISER et al., 2010; SANTOS et al., 2010).

Contudo, neste estudo, ela preveniu erros de magnitude maior que as diferenças causadas pela mudança no comprimento dos cabos, que foram, no máximo, de $0,035 \mathrm{~m}^{3} \mathrm{~m}^{-3}$ (Argissolo-A), quando comparados os cabos de $3 \mathrm{~m}$ e $25 \mathrm{~m}$. Além de prevenir erros de magnitude considerável, o uso da condição de contorno, por evitar desvios bruscos, confere mais chances de a curva obtida ser utilizada em solos semelhantes ao usado na calibração.

\section{CONCLUSÃO}

O uso de curvas de calibração do TDR100, obtidas em laboratório, só é confiável para valores de $\mathrm{K}_{\mathrm{a}}$ medidos no campo, restritos ao intervalo de $\mathrm{K}_{\mathrm{a}}$ usados na elaboração da curva de calibração. A inclusão de medidas de constante dielétrica em água para elaboração da curva de calibração aumenta a acurácia da estimativa do conteúdo de água no campo. Sem esse procedimento e sem verificação de campo das medidas de $\mathrm{K}_{\mathrm{a}}$, erros na estimativa de $\theta$ da ordem de $0,1 \mathrm{~m}^{3} \mathrm{~m}^{-3}$ podem ocorrer facilmente. 


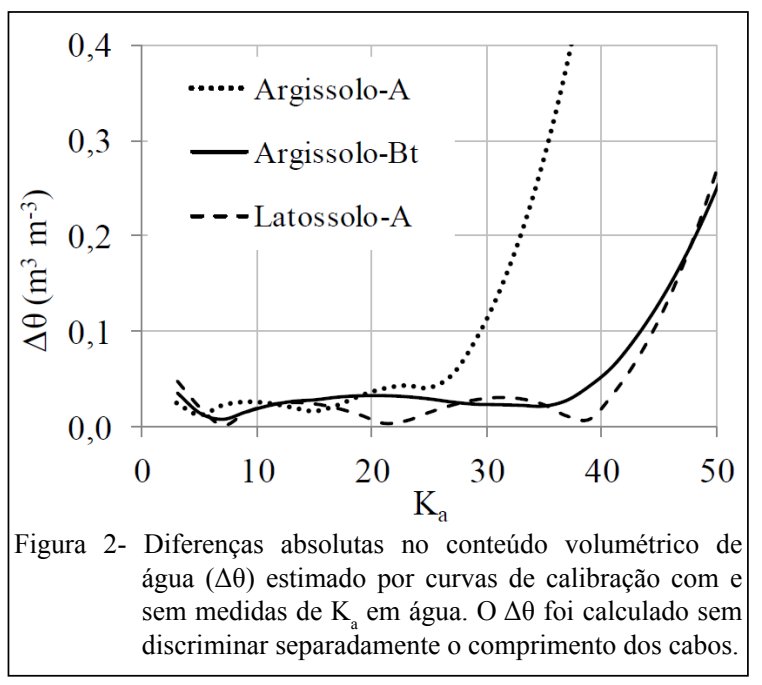

\section{AGRADECIMENTOS}

Os autores agradecem à da Coordenação de Aperfeiçoamento de Pessoal de Nível Superior (CAPES) e ao Conselho Nacional de Desenvolvimento Científico e Tecnológico (CNPq), pelo suporte financeiro (Edital MCT/ CNPq n014/2010 - Universal).

\section{REFERÊNCIAS}

COELHO, E.F. et al. Desempenho de modelos de calibração de guias de onda acopladas a TDR e a multiplexadores em três tipos de solos. Revista Brasileira de Ciência do Solo, v.30, n.1, p.23-30, 2006. Disponível em: <http://www.scielo.br/scielo. php? script $=$ sci_arttext\&pid=S0100-06832006000100003\&lng $=$ pt\&nrm=iso\&tlng=pt>. Acesso em: 13 maio 2014. doi:10.1590/ S0100-06832006000100003.

COELHO, E.F. et al. Sonda de TDR para estimativa da umidade e condutividade elétrica do solo, com uso de multiplexadores. Revista Brasileira de Engenharia Agrícola e Ambiental, v.9, n.4, p.475-480, 2005. Disponível em: <http://www.scielo.br/scielo. php?script $=$ sci_arttext\&pid $=$ S1415-43662005000400006\&lng $=$ pt\&nrm=iso $>$. Acesso em: 13 maio 2014. doi: 110.1590/S141543662005000400006 .

ELAIUY, M. et al. Desenvolvimento e avaliação de sonda de TDR para o manejo racional da água em substratos utilizados na produção de mudas florestais. Revista Ambiente \& Água, v.4, n.1, p.117-131, 2009. Disponível em: <http://www.ambi-agua.net/ seer/index.php/ambi-agua/article/view/188>. Acesso em: 13 maio 2014. doi: 10.4136/1980-993X.

JONES, S.B. et al. Time domain reflectometry measurement principles and applications. Hydrological Processes, v.16, n.1, p.141-153, 2002. Disponível em: < http://onlinelibrary.wiley.com/ doi/10.1002/hyp.513/abstract>. Acesso em: 13 maio 2014.doi: 10.1002/hyp.513.

KAISER, D.R. et al. Dielectric constant obtained from TDR and volumetric moisture of soils in southern Brazil. Revista Brasileira de Ciência do Solo, v.34, n.3, p.649-658, 2010. Disponível em: $<$ http://www.scielo.br/scielo.php?script=sci_arttext\&pid=S01000 $6832010000300006 \& \operatorname{lng}=$ pt\&nrm $=$ iso $\&$ tlng=en $>$. Acesso em: 13 maio 2014. doi:10.1590/S0100-06832010000300006.

SANTOS, M.R.et al. Influência do tipo de amostragem na constante dielétrica do solo e na calibração de sondas de TDR. Revista Brasileira de Ciência do Solo, v.34, n.2, p.299-308, 2010. Disponível em: <http://www.scielo.br/scielo.php?pid=S0100$06832010000200003 \&$ script $=$ sci_arttext $>$. Acesso em: 13 maio 2014. doi: 10.1590/S0100-06832010000200003.

SERRARENS, D. et al. Soil moisture calibration of TDR level probes. Scientia Agricola, v.57, n.2, p.349-354, 2000. Disponível em: $<$ http://www.scielo.br/scielo.php?script=sci_arttext\&pid=S0103$90162000000200024 \& \operatorname{lng}=$ pt\&nrm=iso\&tlng=en>. Acesso em: 13 maio 2014. doi:10.1590/S0103-90162000000200024.

STANGL,R. et al. Field use and calibration of a TDR-based probe for monitoring water content in a high-clay landslide soil in Austria. Geoderma, v.150, n.1-2, p.23-31, 2009. Disponível em: <http:// www.sciencedirect.com/science/article/pii/S0016706109000020>. Acesso em: 13 maio 2014. doi:10.1016/j.bbr.2011.03.031.

TOMMASELLI, J.T.G.; BACCHI, O.O.S. Calibração de um equipamento de TDR para medida da umidade de solos. Pesquisa Agropecuária Brasileira, v.36, n.9, p.1145-1154, 2001. Disponível em: <http://www.scielo.br/scielo.php?pid=S0100204X2001000900008\&script $=$ sci_arttext $>$. Acesso em: 13 maio 2014. doi:10.1590/S0100-204X2001000900008.

TOPP, G.C. et al. Electromagnetic determination of soil water content: measurements in coaxial transmission lines. WaterResourcesResearch, v.16, n.3, p.547-582, 1980. Disponível em: <http://onlinelibrary.wiley.com/doi/10.1029/WR016i003p00574/ abstract>. Acesso em: 13 maio 2014. doi: 10.1029/WR016i003p00574. 